\title{
Effects of transection of cervical sympathetic trunk on cognitive function of traumatic brain injury rats
}

This article was published in the following Dove Press journal:

Neuropsychiatric Disease and Treatment

\author{
Juan Long' \\ Chunjing $\mathrm{He}^{\mathrm{l}}$ \\ Hong $\mathrm{Dai}^{2}$ \\ Xinguo Kang' \\ Jinfeng Zou' \\ Shengli Ye' \\ Qian $\mathrm{Yu}^{2}$ \\ 'Department of Pain, Guizhou Province \\ People's Hospital, Guiyang, People's \\ Republic of China; ${ }^{2}$ Department of \\ Neurology, Guizhou Province People's \\ Hospital, Guiyang, People's Republic of \\ China
}

Objective: To observe the effects of transection of cervical sympathetic trunk (TCST) on the cognitive function of traumatic brain injury (TBI) rats and the potential mechanisms.

Methods: A total of 288 adult male SD rats were divided into 3 groups using a random number table: TBI group $(n=96)$, TBI + TCST group $(n=96)$ and Sham group $(n=96)$. The water maze test was performed before TBI (T0) and at day $1\left(\mathrm{~T}_{1}\right)$, day $2\left(\mathrm{~T}_{2}\right)$, day $3\left(\mathrm{~T}_{3}\right), 1$ week $\left(\mathrm{T}_{4}\right), 2$ weeks $\left(\mathrm{T}_{5}\right), 6$ weeks $\left(\mathrm{T}_{6}\right)$ and 12 weeks $\left(\mathrm{T}_{7}\right)$ after TBI. The levels of $\alpha 1$-adrenergic receptors ( $\alpha 1$-ARs), $\alpha 2$-adrenergic receptors ( $\alpha 2$-ARs), toll-like receptor 4 (TLR-4) and P38 in hippocampi were detected by real-time PCR. Hippocampal P38 expression was assayed by Western blot. The expressions of interleukin-6 (IL-6), tumor necrosis factor (TNF- $\alpha$ ) and brain-derived neurotrophic factor (BDNF) were examined by immunohistochemistry. Noradrenaline (NE) expression in plasma was evaluated by ELISA. The respiratory control ratio (RCR) of brain mitochondria was detected using a Clark oxygen electrode.

Results: TCST effectively improved the cognitive function of TBI rats. TCST significantly inhibited sympathetic activity in the rats and effectively inhibited inflammatory responses. The expression of BDNF at $\mathrm{T}_{1}-\mathrm{T}_{6}$ in TBI+TCST group was higher than that in TBI group $(P<0.05)$. Furthermore, $\mathrm{P} 38$ expression was inhibited more effectively in TBI+TCST group $(P<0.05)$, than in TBI group $(P<0.05)$, and the RCR of the brain was significantly higher in TBI+TCST group than in TBI group $(P<0.05)$.

Conclusions: TCST can enhance cognitive function in TBI rats by inhibiting sympathetic activity, reducing inflammatory responses and brain edema, upregulating BDNF and improving brain mitochondrial function.

Keywords: traumatic brain injury, transection of the cervical sympathetic trunk, sympathetic nervous system, inflammatory response, mitochondrial function

Traumatic brain injury (TBI) is one of the most common diseases with high mortality in humans. ${ }^{1}$ TBI patients often show different degrees of cognitive and behavioral disorders, ${ }^{2,3}$ and clinical treatment and intervention often fail. A series of pathophysiological changes appear after TBI, such as the sympathetic nervous system response to stress, inflammatory response, brain edema, brain hypoxiaischemia and mitochondrial dysfunction. ${ }^{4,5}$ In recent years, the autonomic nervous system has been revealed to play an important role in stress and the inflammatory responses after severe trauma. ${ }^{6}$ The sympathetic nervous system shows hyperexcitability after severe trauma and secretes numerous inflammatory cytokines, thus
Department of Pain, Guizhou Province People's Hospital, No.83 Zhongshan East Road, Guiyang, Guizhou Province,

People's Republic of China

Tel +861 3608538330

Email 777330@I63.com 
resulting in systemic inflammatory response syndrome (SIRS). ${ }^{7}$ Thus, effectively inhibiting stress and inflammatory responses is especially important for improving the prognosis and reducing the mortality of TBI patients. Therefore, exploring favorable treatments that can improve or even completely inhibit these secondary injuries is a significant goal for the treatment of cognitive dysfunction in TBI patients. Stellate ganglion block (SGB) can improve cellular and humoral immune functions and regulate abnormalities in the neuroendocrine system, thus relieving overactivation of the sympathetic nervous system throughout the body. ${ }^{8,9}$ SGB has been widely used for a variety of clinical diseases and has exerted favorable curative effects in recent years. Transection of cervical sympathetic trunk (TCST) of rats is regarded as a satisfactory animal model simulating longterm SGB in humans. ${ }^{10}$ Studies have suggested that unilateral SGB can improve and block the volume of blood flow to the hemibrain and thus protect brain functions. ${ }^{11}$ Studies have shown that SGB exerts a certain improvement effect on cognitive dysfunction, ${ }^{12,13}$ but the mechanism is unknown. Therefore, in this study, early TCST was conducted in rats after TBI to observe its effects on the sympathetic nervous system, inflammatory responses, brain edema and mitochondrial respiratory functions and to analyze the potential mechanism by which TCST improves cognitive functions after TBI.

\section{Materials and methods}

\section{Experimental animals and grouping}

In this experiment, 288 healthy adult naïve male SpragueDawley rats weighing $250-300 \mathrm{~g}$ were provided by the animal laboratory of Third Military Medical University (license No. for animal use: SCXK [Chongqing] 2012-0005). Animal feeding was conducted by the animal laboratory of the Third Military Medical University. The rats were housed in cages with ad libitum access to food and water and divided into the TBI group (TBI group, $\mathrm{n}=96)$, TBI + TCST group (TBI + TCST group, $\mathrm{n}=96)$ and sham operation group (Sham group, $\mathrm{n}=96$ ) using a random number table. All procedures used in this study were in accordance with the National Institutes of Health Guide for the Care and Use of Laboratory Animals and approved by the Institutional Animal Care and Use Committee of the Third Military Medical University. All efforts were made to minimize animal suffering and the number of animals used.

\section{Establishment of models}

Sham group model: After the rat was placed under prone anesthesia, its head was shaved, the iodophor was disinfected, the median incision was made, the periosteum was removed and the left parietal bone was exposed. A dental drill was then used to create a $5 \mathrm{~mm}$ bone window located $1.5 \mathrm{~mm}$ behind the coronal suture and $2.5 \mathrm{~mm}$ adjacent to the midline. The intact periosteum was retained; the rat was then sutured and allowed to continue feeding.

TBI model: This model was constructed using Feeney's weight-dropping impact method. ${ }^{14}$ The pedal hit by the falling body attaches directly to the dura mater (area $4.6 \mathrm{~mm} \times 4.0 \mathrm{~mm}$ ). Before the impact, each rat was anesthetized with an intraperitoneal injection of $10 \%$ chloral hydrate $(300 \mathrm{mg} / \mathrm{kg})$. After the rat was fixed in prone position, its head was shaved and disinfected. The median incision was made to strip the periosteum and expose the left parietal bone. A dental drill was used to drill a bone window (5 $\mathrm{mm}$ in diameter) located $1.5 \mathrm{~mm}$ behind the coronal seam and $2.5 \mathrm{~mm}$ adjacent to the midline to maintain the intact periosteum. The impact bar was placed on the dura mater, and $20 \mathrm{~g}$ weights fell from $25 \mathrm{~cm}$ high. According to $\mathrm{Lu}$ et al, ${ }^{15}$ this method was used to establish a moderate TBI in rats. The experimental TBI model was established successfully.

TCST model: After successful establishment of the TBI model, the skin from the neck was prepared, sterilized with iodine and incised at the median to isolate the left cervical sympathetic nerve. The sympathetic stem was dissociated at $3 \mathrm{~mm}$ superior to the sympathetic ganglia. ${ }^{10}$ After resuscitation from anesthesia, the rats showed typical signs of Horner syndrome, such as ptosis on the same side of cervical sympathetic transection, small eye fission and pupil narrowing, which indicate the successful modeling of cervical sympathetic trunk transection. Thus, the TCST model was successfully established in this experiment.

\section{Behavioral tests}

Twelve rats were randomly selected from each group and subjected to water maze training for 4 days before the models were established. Rats were placed on the platform for $10 \mathrm{~s}$ for adaptation prior to the experiment and then placed randomly in the tank at different quadrant locations. The rats were led to the platform, and recordings were ended after the rats reached the platform and remained there after $10 \mathrm{~s}$ of adaptation. Twelve rats randomly selected from each group were withdrawn from the 
platform before TBI $\left(\mathrm{T}_{0}\right)$ or at day $1\left(\mathrm{~T}_{1}\right)$, day $2\left(\mathrm{~T}_{2}\right)$, day $3\left(\mathrm{~T}_{3}\right), 1$ week $\left(\mathrm{T}_{4}\right), 2$ weeks $\left(\mathrm{T}_{5}\right), 6$ weeks $\left(\mathrm{T}_{6}\right)$ or 12 weeks $\left(\mathrm{T}_{7}\right)$ after TBI and placed in the tank. The target quadrant distance, target quadrant residence time and number of times the rat passed the platform were recorded over 2 mins. Data acquisition was completed using the water maze image supervisory system. After the behavioral indicators were tested at each time point, 6 out of 12 rats were randomly selected for immunohistochemical analysis and brain water content, PCR, ELISA, Western blot and mitochondrial RCR analyses were performed on the brain tissue, hippocampal tissue and blood samples collected from the other 6 rats.

\section{Expressions of IL-6, TNF- $\alpha$ and BDNF in hippocampi by immunohistochemistry}

After the behavioral tests and anesthesia, the thoracic cavities of the selected 6 rats were opened, and a syringe needle was inserted into the apex of the heart. The right auricle was cut and infused rapidly with $100 \mathrm{~mL}$ of physiological saline followed by $4 \%$ paraformaldehyde. The brain was removed, immersed in $4 \%$ paraformaldehyde for $72 \mathrm{hrs}$, dehydrated and embedded in paraffin wax. Then, the brain was sliced into continuous $4-\mu \mathrm{m}$-thick coronal sections. Each section that contained hippocampal tissue was mounted on an anti-slip glass slide that was then baked at $60^{\circ} \mathrm{C}$ for $2 \mathrm{hrs}$. These sections were repaired with antigen repair fluid for 20 mins, incubated and immersed in $3 \% \mathrm{H}_{2} \mathrm{O}_{2}$ for 10 mins to block endogenous peroxidase, and washed with PBS 3 times for 3 mins. The sections were incubated with primary antibodies against interleukin-6 (IL-6) (1:400, Abcam, USA), tumor necrosis factor (TNF- $\alpha)(1: 250$, Abcam, USA), and brain-derived neurotrophic factor (BDNF) $\left(1: 150\right.$, Abcam, USA) at $4{ }^{\circ} \mathrm{C}$ overnight and then washed with PBS 3 times for 30 mins. Polymeric reagents were added, and the sections were incubated at $37^{\circ} \mathrm{C}$ for 15 mins and then washed with PBS 3 times for 2 mins. Samples were stained with DAB coloring reagent and observed under a microscope. The DAB reaction was halted with tap water, and the samples were restained with hematoxylin, returned to blue with tap water, dehydrated until transparent and then mounted. Cells positively expressing IL-6, TNF- $\alpha$ and BDNF were identified based on the detection of yellow or brownish granules in the cytoplasm. The hippocampal tissue sections of 6 rats in each group were observed. Five nonoverlapping fields were randomly observed under a high- power microscope $(10 \times 40$ times $)$ to detect the positive expression of IL- 6 , TNF- $\alpha$ and BDNF. The integrated optical density (IOD) of IL-6 TNF- $\alpha$, BDNF expression was determined by ImagePro Plus 6.0 image analysis software for statistical analysis.

\section{Determination of brain water content}

A cube of the cerebral cortex (approximately $5 \mathrm{~mm} \times 9 \mathrm{~mm} \times 9 \mathrm{~mm} \times 5 \mathrm{~mm}$ ) located near the lesion was dissected, immediately weighed on the electronic balance to obtain the wet weight (WW), dried in a hightemperature drying oven at $105^{\circ} \mathrm{C}$ for $48 \mathrm{hrs}$, and weighed until a constant weight was obtained, ie, the dry weight (DW). The brain water content was calculated based on the Elliott formula as follows: brain water content $(\%)=$ (WW - DW) $/ \mathrm{WW} \times 100 \%$.

\section{RNA extraction and real-time PCR}

Total RNA was extracted from hippocampal cells using TRIzol and reverse transcribed into cDNA. The following primers were used: P38 (sense: 5'-CGGCTTGCTCATGT CCTCAGAAC-3', antisense: 5-'GGAGGGCGGCTGCAC ATACAC-3'); toll-like receptor 4 (TLR-4) (sense: 5'AGCCCTGTTGGATGGAAAAGC-3', antisense: 5'-GGGT TTTAGGCGCAGAGTTTTG-3'); $\alpha 1$-adrenergic receptors (a1-ARs) (sense: 5'-TGGGCCATCTCCGCGCTG-3', antisense: 5'-GCCCGGTTGGTGACGAAATC-3'); $\alpha 2$-adrenergic receptors ( $\alpha 2$-ARs) (sense: 5'-TTCTGTGCCTTC GCCGGTCTTCC-3', antisense: 5'-TCAGGGAGGGGCCG TCTTAAAG-3'). Each reaction was performed in triplicate using Maxima SYBR Green/ROX qPCR Master Mix (2×) according to the manufacturer's protocol. The PCR conditions were as follows: predenaturation at $96^{\circ} \mathrm{C}$ for 6 mins; 40 cycles of denaturation at $96^{\circ} \mathrm{C}$ for $30 \mathrm{~s}$, annealing at $57^{\circ} \mathrm{C}$ for $30 \mathrm{~s}$, and extension at $72^{\circ} \mathrm{C}$ for $30 \mathrm{~s}$; and a final extension step at $72^{\circ} \mathrm{C}$ for 10 mins. The $2^{-\triangle \triangle \mathrm{CT}}$ value was calculated.

\section{Assessing the expression of P38 by Western blot}

RIPA lysis buffer and phenylmethylsulfonyl fluoride (PMSF) were mixed at a 100:1 volume ratio to fully lyse the tissue; the resulting mixture was centrifuged at $4^{\circ} \mathrm{C}$ at $12,000 \mathrm{rpm}$ for $15 \mathrm{mins}$, and the supernatant was then collected. The protein concentration of the sample was detected using the BCA protein assay kit. After vertical electrophoresis, the samples were transferred for $1.5 \mathrm{hrs}$, sealed for $2 \mathrm{hrs}$, incubated with the primary 
antibody (Abcam, 1:500) at $4^{\circ} \mathrm{C}$ overnight, and then incubated with the secondary antibody $(1: 1000)$ at room temperature for $1 \mathrm{hr}$. Chemiluminescence was performed with enhanced chemiluminescence (ECL) luminous liquid, and films were used for exposure.

\section{Assessing the expression of serum noradrenaline (NE) by ELISA}

Rat peripheral venous blood $(1 \mathrm{ml})$ was collected, incubated at room temperature for $2 \mathrm{hrs}$ and centrifuged at $2000 \times \mathrm{g}$ for $20 \mathrm{mins}$; the supernatant was then collected to detect the level of serum NE with the ELISA kit (USA R\&D) based on the kit instructions.

\section{Determination of the brain mitochondrial respiratory control ratio ( $R C R$ )}

The brain tissue in the lesioned area was dissolved in the separation medium to obtain the tissue homogenate, which was centrifuged at $12,000 \times \mathrm{g}$ at $4^{\circ} \mathrm{C}$ for $10 \mathrm{mins}$ 2 times; the precipitant contained the mitochondria. The mitochondria $(2 \mathrm{mg})$ were added to $3 \mathrm{~mL}$ of reaction medium $(225 \mathrm{mmol} / \mathrm{L}$ mannitol, $70 \mathrm{mmol} / \mathrm{L}$ sucrose, 1 $\mathrm{mmol} / \mathrm{L}$ EDTA, $0.1 \% \mathrm{BCA}, 10 \mathrm{mmol} / \mathrm{L}$ potassium phosphate, $\mathrm{pH} 7.4$, temperature $25^{\circ} \mathrm{C}$ ) and stirred until the basic concentration was stable $(240 \mathrm{nmol} / \mathrm{mL})$. Then, 20 $\mu \mathrm{L}$ of the mitochondrial suspension (approximately $2 \mathrm{mg}$ ) was incubated with $20 \mu \mathrm{L}$ of substrate (disodium succinate $4 \mathrm{mmol} / \mathrm{L}$ ) for 2 mins. Clark's oxygen electrode method was then used to detect the respiratory oxygen quotient IV (R4). ${ }^{16}$ Then, $20 \mu \mathrm{L}$ of adenosine diphosphate (ADP, $50 \mathrm{mmol} / \mathrm{L}$ ) was added, and the respiratory oxygen quotient III (R3) was measured. The mitochondrial RCR was calculated using the following equation: $\mathrm{RCR}=\mathrm{R} 3 / \mathrm{R} 4$.

\section{Statistical analysis}

Statistical analysis was conducted with SPSS16.0. The data are all expressed as $\pm \mathrm{S}$. Comparisons among groups were analyzed with one-way ANOVA; multiple comparisons were analyzed with least square difference (LSD) tests. $P<0.05$ suggested that differences were statistically significant.

\section{Results}

\section{Analysis of the behavioral effects of TCST on $\mathrm{TBI}$ rats}

Behavioral indexes of rats in the three groups were measured by the water maze test. Compared with those in Sham group, rats in TBI group exhibited an increase in the target quadrant distance at $\mathrm{T}_{1-4}(P<0.05)$ (Figure 1A) and in the target quadrant time at $\mathrm{T}_{1-4}(P<0.05)$ (Figure 1B). Furthermore, the number of platform crosses at $\mathrm{T}_{1-6}$ was lower in TBI group than in Sham group $(P<0.05)$ (Figure 1C). There were no significant differences in behavioral indicators among TBI + TCST group, Sham group or TBI group at any time point.

\section{Effects of TCST on the sympathetic nervous system of $\mathrm{TBI}$ rats}

Compared to that in Sham group, the plasma NE level in TBI group at $\mathrm{T}_{1-5}$ was obviously higher $(P<0.05)$; however, there were no significant differences in the plasma NE levels between TBI+TCST group and Sham group and TBI group (Figure 2A). Hippocampal $\alpha 1$-ARs expression was significantly lower at $\mathrm{T}_{1-5}$ in TBI group and TBI+TCST group compared with that in Sham group $(P<0.05)$; hippocampal $\alpha 1$-ARs expression was obviously lower at $\mathrm{T}_{1-3}$ in TBI group than in TBI+TCST group $(P<0.05)$ (Figure 2B). Hippocampal $\alpha 2$-ARs expression was markedly lower at $T_{1-5}$ in TBI group and TBI+TCST group than in Sham group $(P<0.05)$, and hippocampal $\alpha 2$-ARs expression was lower at $\mathrm{T}_{1-3}$ in TBI group than in TBI+TCST group $(P<0.05)$ (Figure $2 \mathrm{C}$ ).
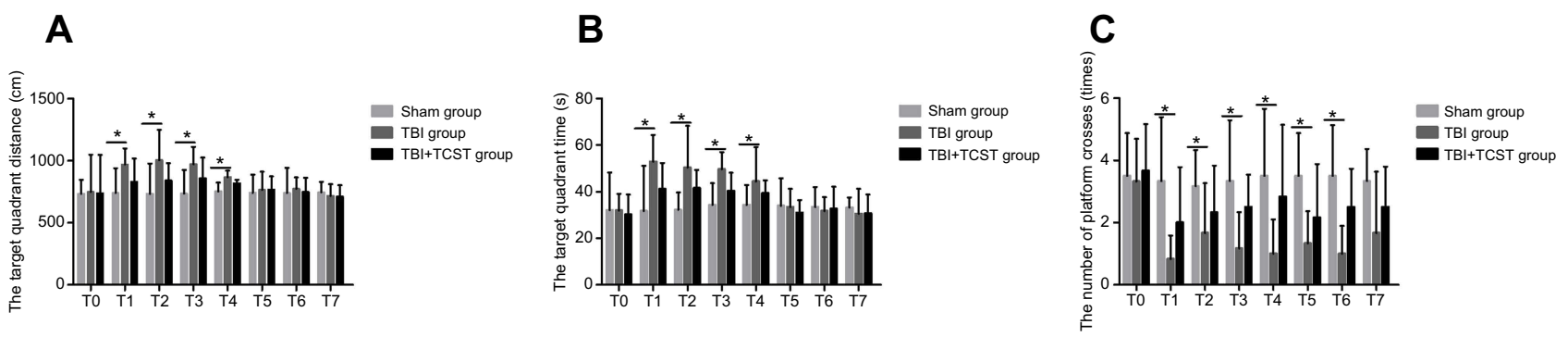

Figure I The behavioral test results of rats in all groups. (A) Comparison of the distances in target quadrants at different time points in rats of all groups. (B) Comparison of the times in target quadrants at different time points in rats of all groups. (C) Comparison of the number of times the rats crossed the platform in all groups. All data are expressed as the mean \pm standard deviation. Six rats per group; Sham group versus TBI group ${ }^{*} p<0.05$, Sham group versus TBI+TCST group * $p<0.05$, TBI group versus TBI + TCST group $* p<0.05$. 

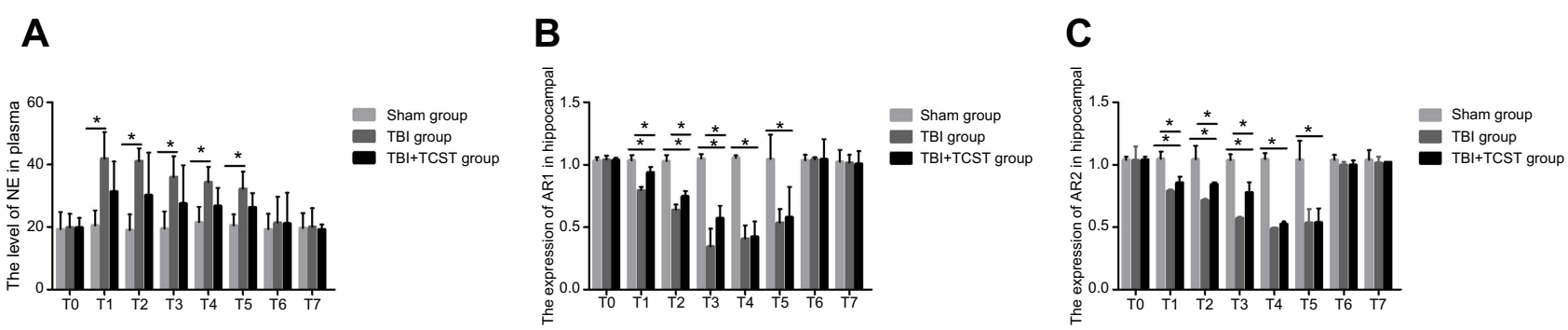

Figure 2 The effects of TCST on the sympathetic nervous system of TBI rats. (A) Comparison of plasma NE levels in rats of each group at each time point. (B) Comparison of hippocampal ARI expression in rats of each group at each time point (2B). (C) Comparison of hippocampal AR2 expression in rats of each group at each time point. All data are expressed as the mean \pm standard deviation. Six rats per group; Sham group versus TBI group $* p<0.05$, Sham group versus TBI + TCST group $* p<0.05$, TBI group versus TBI+TCST group $*_{p}<0.05$.

\section{Analysis of the effects of TCST on the inflammatory responses of hippocampi in TBI rats}

Compared with that in Sham group and TBI+TCST group, the expression of hippocampal IL-6 was markedly increased in TBI group at $\mathrm{T}_{1-6}(P<0.05)$. The expression of IL-6 was higher in TBI+TCST group than in Sham group at $\mathrm{T}_{1-4}$ $(P<0.05)$ (Figures 3 and 5A). The expression of hippocampal TNF- $\alpha$ was significantly higher in TBI group at $T_{1-6}$ than in Sham group and TBI+TCST group, and the expression of TNF- $\alpha$ was higher in TBI+TCST group than in Sham group at $\mathrm{T}_{1-4}(P<0.05)$ (Figures 4 and $\left.5 \mathrm{~B}\right)$.

\section{Brain water content}

The brain water content in TBI group at $\mathrm{T}_{1-4}$ was obviously higher than that in Sham group and TBI + TCST group $(P<0.05)$. No significant differences in the brain water contents of Sham group and TBI+TCST group were observed at any time point (Table 1).

\section{Changes in the expression of hippocampal BDNF in rats from each group}

Compared with that in Sham group and TBI+TCST group, the expression of hippocampal BDNF was significantly
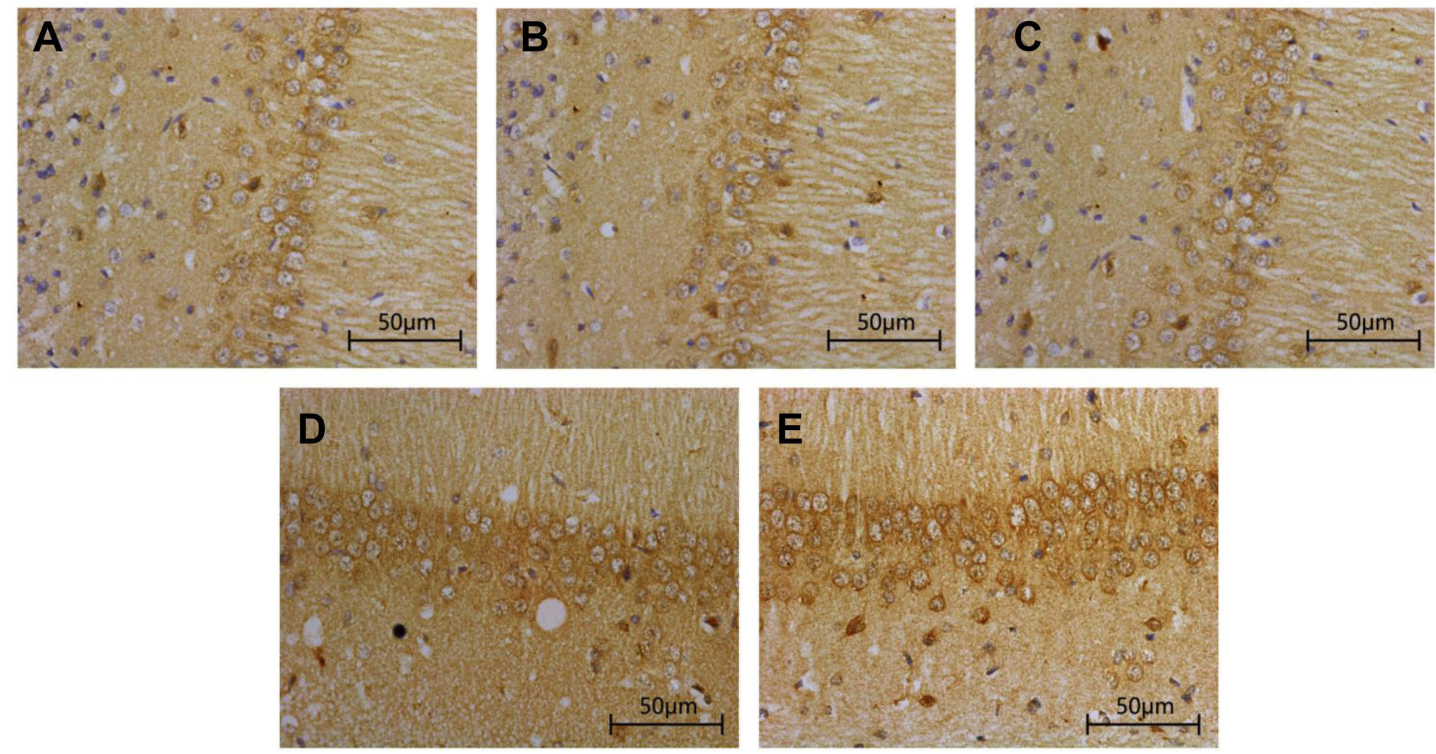

Figure 3 Effects of TCST on the inflammatory responses of hippocampi in TBI rats. Immunohistochemical staining of IL-6 in the hippocampi of TBI rats after TCST (400×s). A-E: (A-C) The visual fields at time $\mathrm{T}_{0}$ in Sham group, TBI group and TBI+TCST group under an optical microscope. Minor hippocampal IL-6 expression was observed at $\mathrm{T}_{0}$ in the three groups. (D) The visual fields at all time points except for $\mathrm{T}_{5-7}$ in TBI+TCST group under an optical microscope. The expression of IL-6 was increased in TBI +TCST group compared to that in the other groups. (E) The visual fields at all time points except for $\mathrm{T}_{7}$ in TBI group under an optical microscope. The expression of IL-6 was increased more significantly in TBI group than in the other two groups. Six rats per group. 

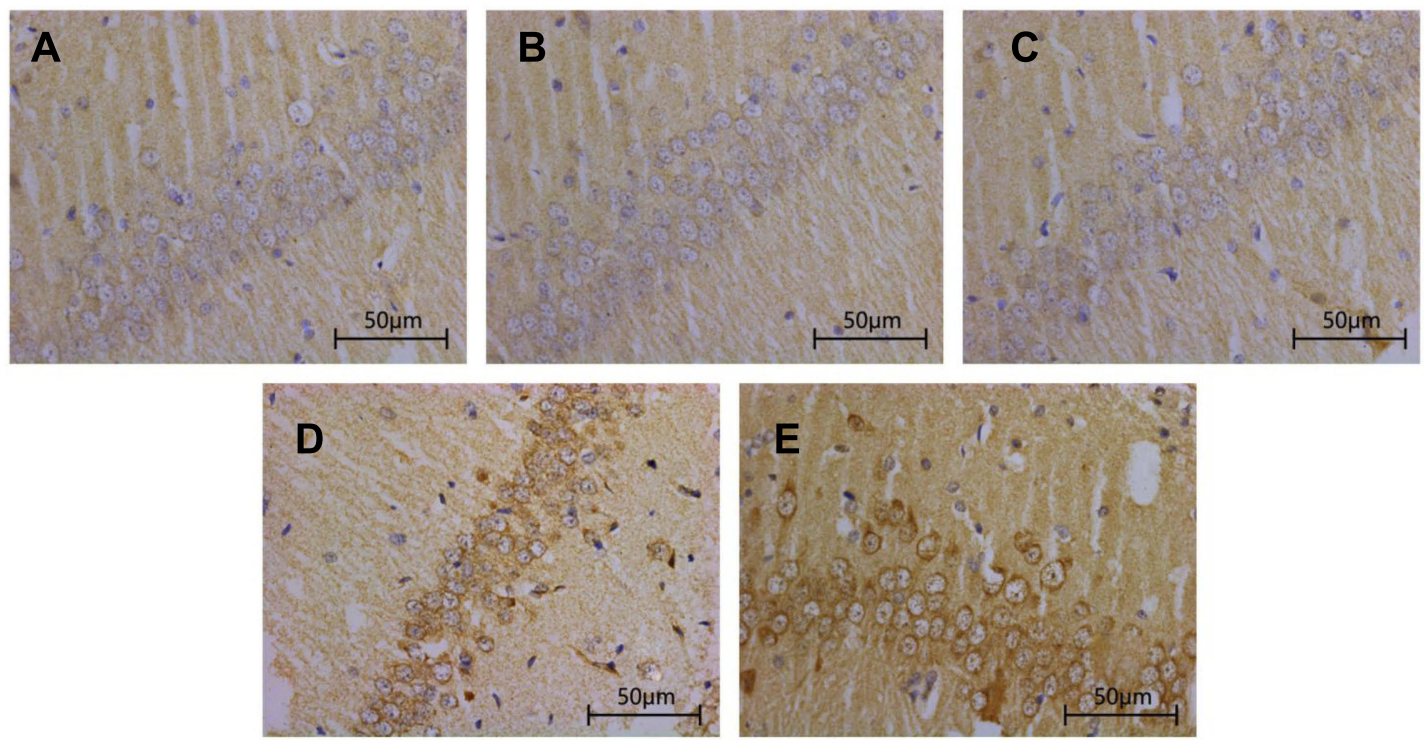

Figure 4 The effects of TCST on the inflammatory responses of hippocampi in TBI rats. Immunohistochemical staining of TNF- $\alpha$ in the hippocampi of TBI rats after TCST (400x). A-E: (A-C) The visual fields at time $\mathrm{T}_{0}$ in Sham group, TBI group and TBI+TCST group under an optical microscope. Minor hippocampal TNF- $\alpha$ expression was observed at $T_{0}$ in the three groups. (D) The visual fields at all time points except for $T_{5-7}$ in TBI+TCST group under an optical microscope. The expression of TNF- $\alpha$ was increased in TBI+TCST group compared to that in the other groups. (E) The visual fields at all time points except for $\mathrm{T}_{7}$ in TBI group under an optical microscope. The expression of TNF- $\alpha$ was increased more significantly in TBI group than in the other two groups. Six rats per group.

A

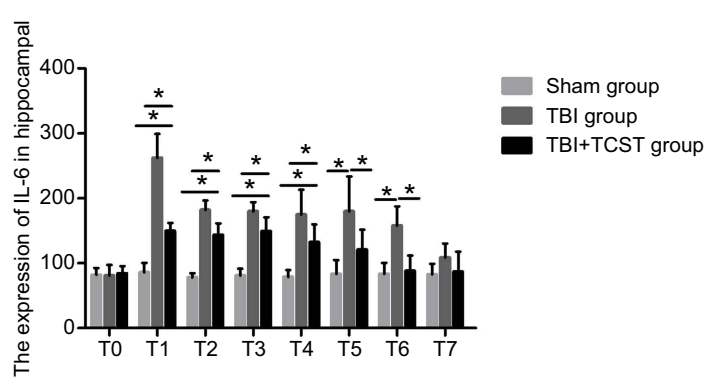

B

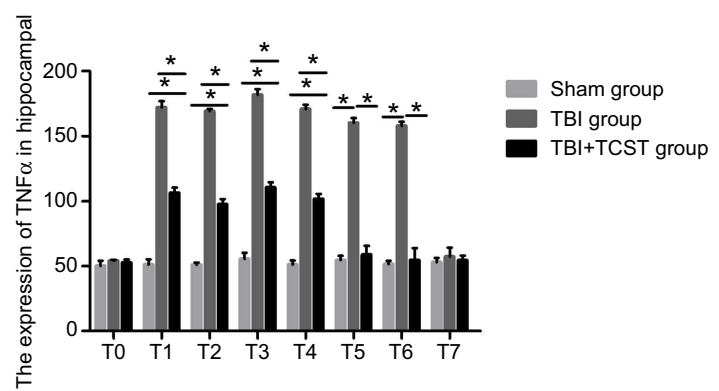

Figure 5 The effects of TCST on the inflammatory responses of hippocampi in TBI rats. Comparison of hippocampal IL-6 (A) and TNF- $\alpha$ (B) expression in rats of each group at each time point. All data are expressed as the mean \pm standard deviation. Six rats per group; Sham group versus TBI group $* p<0.05$, Sham group versus TBI+TCST group $* p<0.05, \mathrm{TBI}$ group versus TBI+TCST group $*_{p}<0.05$.

Table I Comparison of brain water contents in rats from each group at each time point $(g, \pm S D)$

\begin{tabular}{|l|l|l|l|l|l|l|l|l|}
\hline Group & $\mathbf{T}_{\mathbf{0}}$ & $\mathbf{T}_{\mathbf{1}}$ & $\mathbf{T}_{\mathbf{2}}$ & $\mathbf{T}_{\mathbf{3}}$ & $\mathbf{T}_{\mathbf{4}}$ & $\mathbf{T}_{\mathbf{5}}$ & $\mathbf{T}_{\mathbf{6}}$ & $\mathbf{T 7}$ \\
\hline $\begin{array}{l}\text { Sham } \\
\text { group }\end{array}$ & $0.746 \pm 0.0477$ & $0.73 \mathrm{I} \pm 0.034$ & $0.747 \pm 0.049$ & $0.735 \pm 0.042$ & $0.754 \pm 0.025$ & $0.737 \pm 0.037$ & $0.744 \pm 0.057$ & $0.757 \pm 0.035$ \\
TBI group & $0.733 \pm 0.0179$ & $0.895 \pm 0.060^{\mathrm{ab}}$ & $0.825 \pm 0.048^{\mathrm{ab}}$ & $0.826 \pm 0.05 \mathrm{I}^{\mathrm{ab}}$ & $0.822 \pm 0.035^{\mathrm{ab}}$ & $0.813 \pm 0.025$ & $0.786 \pm 0.013$ & $0.767 \pm 0.004$ \\
TBI & $0.748 \pm 0.748$ & $0.768 \pm 0.04 \mathrm{I}$ & $0.74 I \pm 0.043$ & $0.745 \pm 0.0480$ & $0.749 \pm 0.054$ & $0.75 I \pm 0.093$ & $0.767 \pm 0.049$ & $0.75 \mathrm{I} \pm 0.037$ \\
+ TCST & & & & & & & & \\
group & & & & & & & & \\
F value & 0.259 & 17.240 & 4.974 & 5.664 & 5.239 & 2.330 & 1.188 & 0.364 \\
P-value & 0.776 & 0.000 & 0.027 & 0.019 & 0.023 & 0.140 & 0.338 & 0.702 \\
\hline
\end{tabular}

Notes: The brain water content in TBI group at $\mathrm{T}_{1-4}$ was obviously higher than that in Sham group and TBI+TCST group. No significant differences were observed in brain water content at any time point when Sham group was compared with TBI+TCST group. Six rats per group; Sham group versus TBI group ${ }^{a} \mathrm{P}<0.05$, TBI group versus $\mathrm{TBI}$ + TCST group ${ }^{\mathrm{b}}<0.05$. 
lower in TBI group at $\mathrm{T}_{1-7}(P<0.05)$. The expression of BDNF in TBI+TCST group at $\mathrm{T}_{1-6}$ was lower than that in Sham group $(P<0.05)$ (Figures 6 and 7 ).

\section{Assessing the expression of TLR-4 and P38 mRNA by RT-PCR and the expression of $\mathrm{P} 38$ by WB}

The RT-PCR results showed that compared with that in Sham group and TBI+TCST group, the expression of hippocampal TLR-4 was obviously higher in TBI group at $\mathrm{T}_{1-6}(P<0.05)$. TLR-4 expression was also higher in TBI+TCST group at $\mathrm{T}_{1-6}$ than in Sham group $(P<0.05)$ (Figure $8 \mathrm{~A})$. The expression of hippocampal P38 mRNA was obviously higher in TBI group at $\mathrm{T}_{1-6}$ than in Sham group and TBI+TCST group $(P<0.05)$, and the expression of P38 mRNA was higher in TBI+TCST group than in Sham group at $\mathrm{T}_{1-4}(P<0.05)$ (Figure $8 \mathrm{~B}$ ). Western blot showed that the hippocampal expression of the P38 protein was markedly higher in TBI group than in Sham group and TBI+TCST group at $\mathrm{T}_{1-6}(P<0.05)$. The protein expression of $\mathrm{P} 38$ was higher in TBI+TCST group than in Sham group at $\mathrm{T}_{1-4}(P<0.05)$ (Figure 9).

\section{Changes in mitochondrial respiratory function}

Compared with those in Sham group and TBI+TCST group, the mitochondrial respiratory function was obviously reduced in TBI group at $\mathrm{T}_{1-6}(P<0.05)$. The mitochondrial respiratory function was also lower in TBI + TCST group than in Sham group at $\mathrm{T}_{1-5}(P<0.05)$ (Figure $10)$, as shown by the measurement of respiratory function with Clark's oxygen electrode.

\section{Discussion}

A cervical sympathetic block can improve local tissue hypoxia-ischemia and dysbolism by dilating blood vessels in its control area, resulting in better protection from cerebral ischemia-reperfusion injury and a reduction in the tone of the sympathetic nerve center in the hypothalamus caused by stress. ${ }^{10,15}$ TBI patients undergo TCST, which significantly increases plasma calcitonin gene-related peptide (CGRP) expression while obviously reducing NF-kBp65 expression. ${ }^{9}$ In our studies, early after TBI, TCST improved the cognitive function of rats after TBI.

When the body is in a stressed state, the central and peripheral sympathetic nervous systems are activated to release NE, which can cause the release of inflammatory cytokines by binding to adrenergic receptors on nerve cells. ${ }^{10,17}$ Excessive concentrations of NE are also associated with the level of brain injury and have been suggested to affect the prognosis of patients. ${ }^{3}$ Similarly, TLR4 after TBI can mediate inflammatory responses by increasing the release of damage-related molecules from
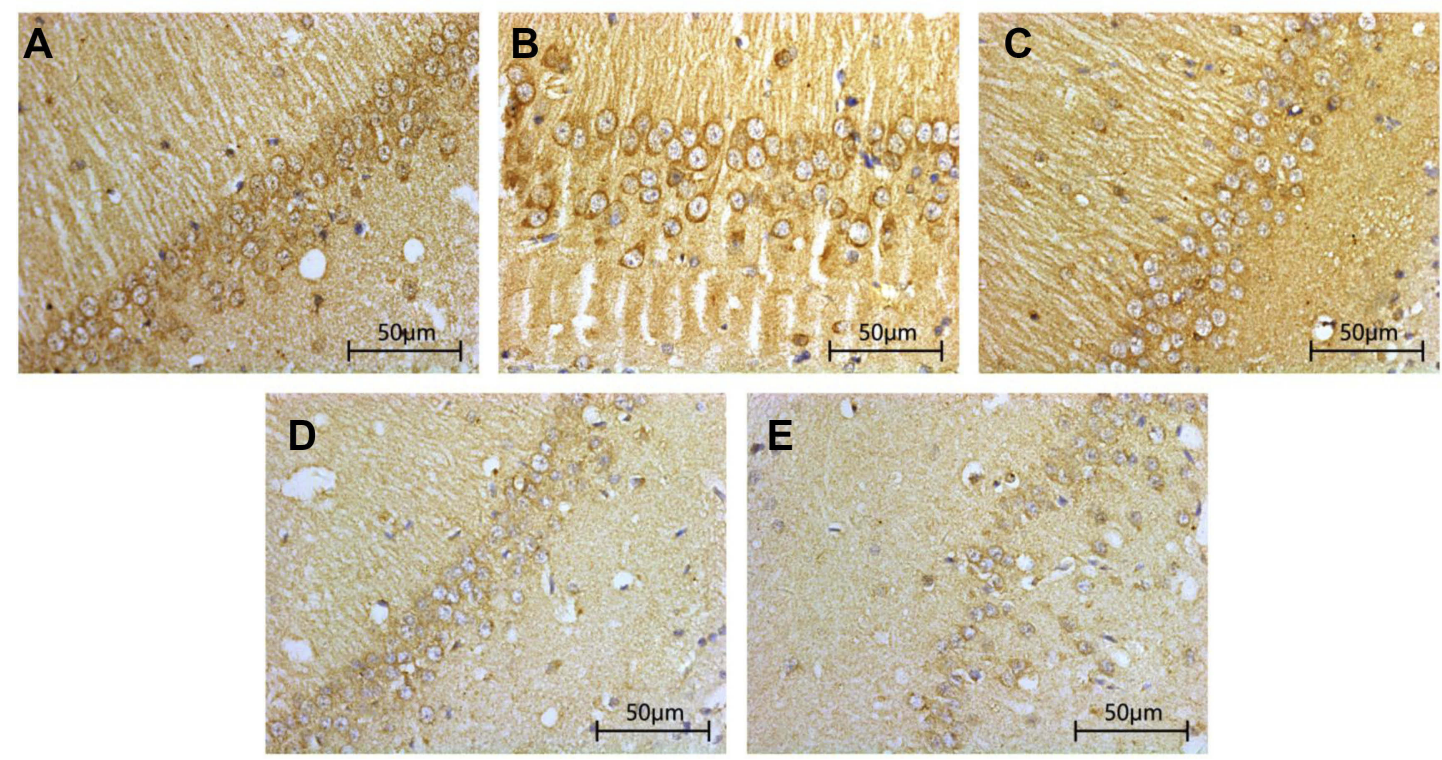

Figure 6 Changes in the hippocampal expression of BDNF in rats of each group. Immunohistochemical staining of BDNF in the hippocampi of TBI rats after TCST ( $\times 400)$. A-E: (A-C) The visual fields at time $T_{0}$ in Sham group, TBI group and TBI+TCST group under an optical microscope. The expression of BNDF was increased at $\mathrm{T}_{0}$ in the three groups. (D) The visual fields at all time points except for $\mathrm{T}_{7}$ in TBI+TCST group under an optical microscope. The expression of BNDF was increased in TBI+TCST group compared to that in the other two groups. (E) The visual fields at all time points in TBI group under an optical microscope. The expression of BNDF was increased more significantly in $\mathrm{TBI}$ group than in the other two groups. Six rats per group. 


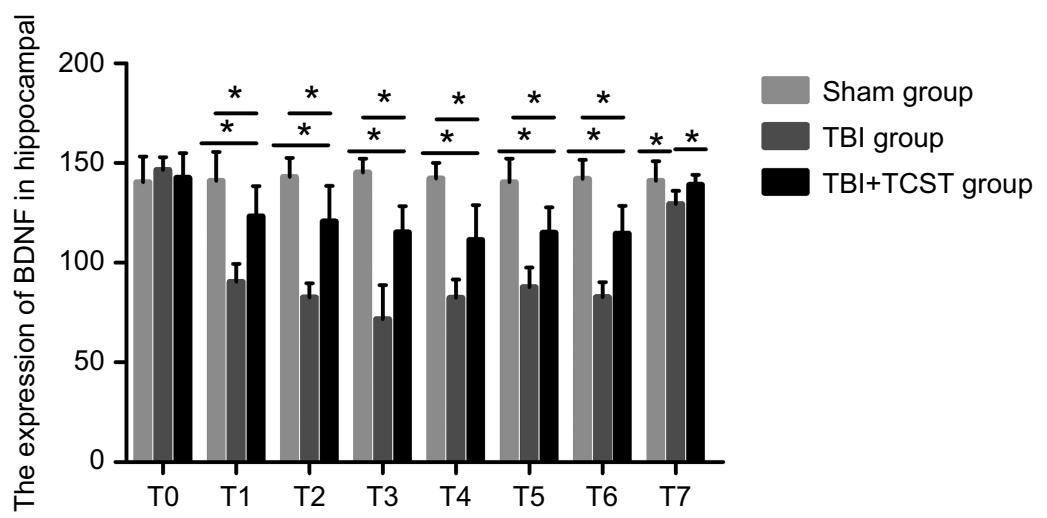

Figure 7 Changes in the hippocampal expression of BDNF in rats of each group. Comparison of hippocampal BNDF expression in rats of each group at each time point. All data are expressed as the mean \pm standard deviation. Six rats per group; Sham group versus TBI group $* p<0.05$, Sham group versus TBI+TCST group $* p<0.05$, TBI group versus TBI+TCST group ${ }^{*} p<0.05$

A

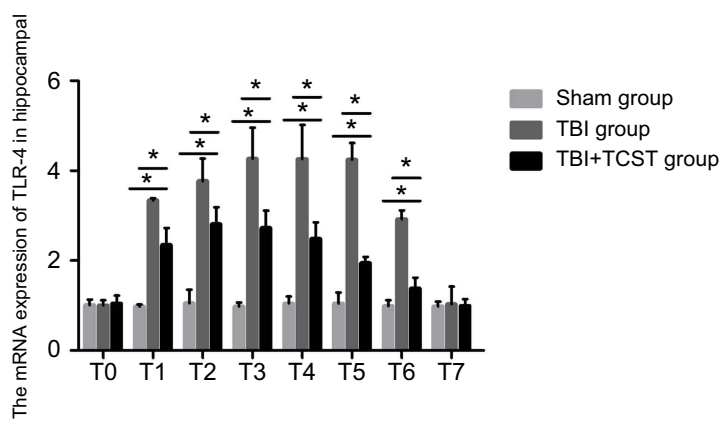

B

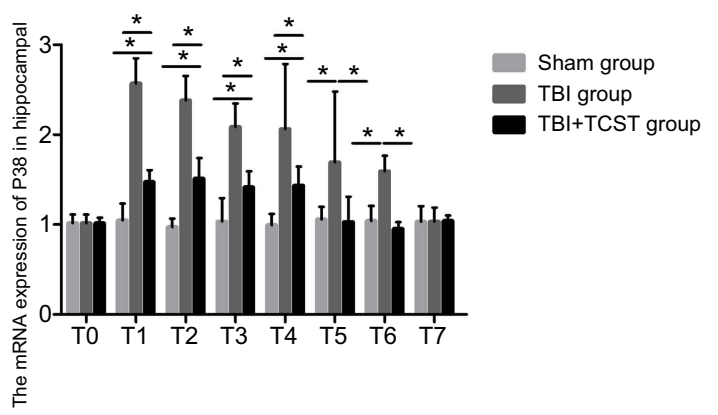

Figure 8 Analysis of TLR-4 and P38 mRNA expression by RT-PCR. Comparison of TLR-4 mRNA levels in rats of each group at each time point (A). Comparison of P38 mRNA levels in rats of each group at each time point (B). All data are expressed as the mean \pm standard deviation. Six rats per group; Sham group versus TBI group * $p<0.05$, Sham group versus TBI+TCST group $* p<0.05$, TBI group versus TBI+TCST group $* p<0.05$.

A

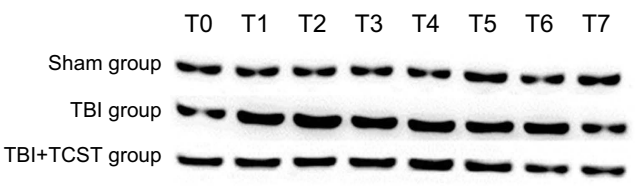

B

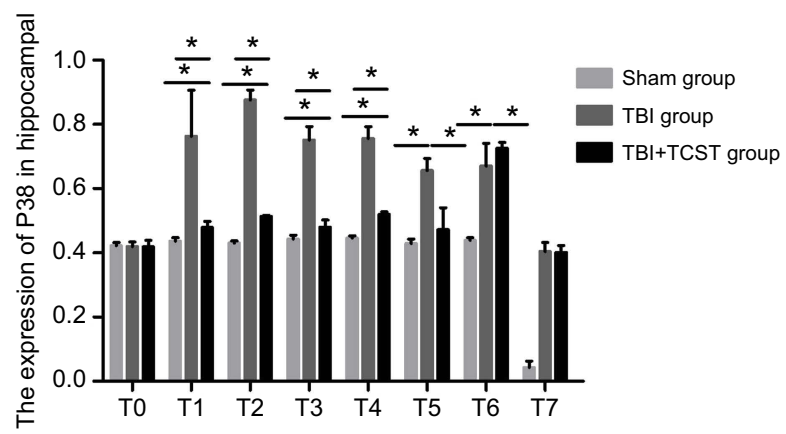

Figure 9 Analysis of P38 mRNA expression by WB. P38 expression was notably increased in the hippocampi of TBI group at $\mathrm{T}_{\mathrm{I}-6}$ and significantly higher than that in TBI +TCST group; P38 expression in TBI+TCST group was still substantially higher than that in Sham group. P38 expression was not statistically significant different among the three groups at $\mathrm{T}_{7}(\mathbf{A})$. Comparison of the $\mathrm{P} 38$ protein expression among rats of each group at each time point (B). All data are expressed as the mean \pm standard deviation. Six rats per group; Sham group versus TBI group $*_{p}<0.05$, Sham group versus TBI+TCST group $*_{p}<0.05$, TBI group versus TBI+TCST group $*_{p}<0.05$.

injured brain tissues. ${ }^{18,19}$ The chemotactic factors of these inflammatory mediators also increase the number of inflammatory cells and immune cell infiltration. The excessive release of inflammatory factors leads to inflammatory cascade responses and further damages the brain tissue. $^{20-22}$ 


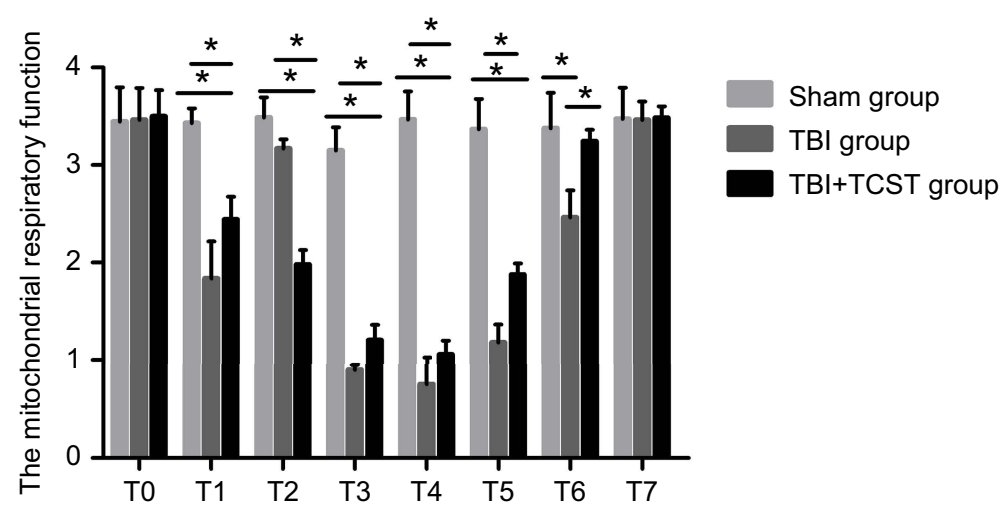

Figure 10 Comparison of the mitochondrial respiratory functions of rats of each group at each time point. All data are expressed as the mean \pm standard deviation. Six rats per group; Sham group versus TBI group $*_{p}<0.05$, Sham group versus TBI+TCST group $* p<0.05$, TBI group versusTBI+TCST group * $p<0.05$.

Our studies revealed that hippocampal NE expression was significantly increased in TBI group at $T_{1-5}$ after TBI, and the hippocampal expression of TLR-4 was obviously increased in TBI group and TBI+TCST group at $\mathrm{T}_{1-6}$, suggesting that TBI could cause sympathetic tonus. Increased hippocampal NE expression is indicative of the occurrence of inflammatory responses; however, we found that TCST treatment can inhibit the sympathetic nervous system tension after TBI and reduce plasma NE levels, thereby reducing the release of IL-6 and TNF- $\alpha$ in the hippocampus and effectively reducing the post-TBI inflammatory response and preventing further brain damage. Excessive inflammatory response after TBI can aggravate brain damage, edema and cell death, thereby aggravating secondary brain injury. ${ }^{21-23}$ Our study showed that the inflammatory response was well controlled after TCST and that brain edema was further improved. Noradrenergic receptor activation in the prefrontal cortex of rats increases the level of arousal and can improve cognitive function. ${ }^{24}$ Hilakivi et al found that AR1 antagonists can prolong the sleep time of cats, ${ }^{25}$ while AR1 agonists shorten their sleep time and maintain the awakening state. In our study, the expression of AR1 and AR2 in the rat hippocampus was reduced to some extent immediately after TBI and upregulated after TCST treatment and 3 days after injury. These findings indicate that TCST intervention after TBI can effectively control the inflammatory response and improve cerebral edema in the acute phase and even the subacute phase after craniocerebral injury, which can improve the prognosis of TBI

Studies have shown that TBI-induced mitochondrial damage can be significantly inhibited in TBI rats treated with the P38 inhibitor SB203580. ${ }^{26}$ In a cerebral ischemic injury study, P38 was shown to be closely related to mitochondrial dysfunction; therefore, inhibition of P38 will provide brain neuron protection. ${ }^{27}$ TCST was able to inhibit the expression of $\mathrm{P} 38$ at $\mathrm{T}_{1-6}$ after injury. Although the expression of P38 was higher in TBI+TCST group than in Sham group at $\mathrm{T}_{1-4}$, the RCR was obviously lower in TBI group than in TBI+TCST group at $\mathrm{T}_{1-6}$, suggesting that TCST can improve the respiratory function of mitochondria by inhibiting the sympathetic tone, inflammatory responses and the expression of P38, as demonstrated in our studies.

Produced by neurons and glial cells, BDNF, a neurotrophic factor, can regulate the development and maturation of the nervous system, maintain proteins involved in nerve functions, promote the rebirth of nervous processes and the formation of new synapses around ischemic foci and in normal brain tissue, and block neuronal apoptosis by a variety of mechanisms after brain injury, thus showing obvious protection of hypoxicischemic neurons during brain injury. ${ }^{28}$ In our experiment, although the expression of BDNF in TBI+TCST group was lower than that in Sham group at $T_{1-6}$, it was obviously higher than that in TBI group at $\mathrm{T}_{1-7}$, suggesting that TCST can improve TBI by upregulating the expression of BDNF, and the duration of this brain protection can last up to 12 weeks after TBI.

The experimental behavioral results demonstrated that the target quadrant distance of rats in TBI+TCST group was shorter than that in TBI group at $\mathrm{T}_{1-4}$. Furthermore, the target quadrant time was also shorter at $\mathrm{T}_{1-4}$ in $\mathrm{TBI}$ + TCST group than in TBI group, and the number of times the rats passed the platform was greater at $T_{1-6}$ in TBI 
+TCST group than in TBI group. However, no obvious differences were observed in the above 3 indexes between TBI+TCST group and Sham group. These results suggest that TCST can improve cognitive dysfunction to a certain extent after TBI.

In summary, TCST can improve the cognitive dysfunction of rats after TBI. This effect may be exerted via inhibition of the sympathetic nerve tone, thus inhibiting inflammatory responses, reducing brain edema and improving brain mitochondrial respiratory function, and upregulating the expression of BDNF. The effects of TCST on the cognitive function of rats from 1 day to 12 weeks after TBI were observed and analyzed in this study. The long-term cognitive function and specific mechanisms, however, need to be studied further.

\section{Acknowledgment}

This study is financially supported by the Doctoral Scientific Fund Project of Guizhou Provincial Peoples' Hospital (GZSYBS[2015]06) and Youth Scientific Fund Project of Guizhou Provincial Peoples’ Hospital (GZSYQN[2016]04).

\section{Disclosure}

The authors have no conflicts of interest to declare in this work.

\section{References}

1. Hiebert JB, Shen Q, Thimmesch AR, Pierce JD. Traumatic brain injury and mitochondrial dysfunction. Am J Med Sci. 2015;350 (2):132-138. doi:10.1097/MAJ.0000000000000506

2. Xiao H, Yang Y, Xi JH, Chen ZQ. Structural and functional connectivity in traumatic brain injury. Neural Regen Res. 2015;10 (12):2062-2071. doi:10.4103/1673-5374.172328

3. Sun HY, Li Q, Chen XP, Tao LY. Mismatch negativity, social cognition, and functional outcomes in patients after traumatic brain injury. Neural Regen Res. 2015;10(4):618-623. doi:10.4103/1673-5374.155437

4. Algattas H, Huang JH. Traumatic brain injury pathophysiology and treatments: early, intermediate, and late phases post-injury. Int $J \mathrm{Mol}$ Sci. 2013;15(1):309-341. doi:10.3390/ijms15010309

5. Di Battista AP, Rhind SG, Hutchison MG, et al. Inflammatory cytokine and chemokine profiles are associated with patient outcome and the hyperadrenergic state following acute brain injury. J Neuroinflammation. 2016;13:40. doi:10.1186/s12974-016-0500-3

6. Yang C, Yan J, Wang HY, et al. Effects of bilateral adrenalectomy on the innate immune responses following trauma in rats. Injury. 2011;42 (9):905-912.

7. Steinman L. Elaborate interactions between the immune and nervous systems. Nat Immunol. 2004;5(6):575-581. doi:10.1038/ni1078

8. Shin JH, Kim DW, Yang JY, Lee WI. Efficacy of stellate ganglion block in cholinergic urticaria with acquired generalized hypohidrosis. Korean J Pain. 2012;25(4):278-280. doi:10.3344/kjp.2012.25.4.278

9. Yang X, Shi Z, Li X, Li J. Impacts of stellate ganglion block on plasma NF- $\mathrm{kB}$ and inflammatory factors of TBI patients. Int J Clin Exp Med. 2015;8(9):15630-15638.
10. Hu N, Wu Y, Chen BZ, Han JF, Zhou MT. Protective effect of stellate ganglion block on delayed cerebral vasospasm in an experimental rat model of subarachnoid hemorrhage. Brain Res. 2014;1585:63-71. doi:10.1016/j.brainres.2014.08.012

11. Kim EM, Yoon KB, Lee JH, Yoon DM, Kim DH. The effect of oxygen administration on regional cerebral oxygen saturation after stellate ganglion block on the non-blocked side. Pain Physician. 2013;16(2):117-124.

12. Chun-Jing H, Shan O, Guo-Dong L, Hao-Xiong N, Yi-Ran L, YaPing F. Effect of cervical sympathetic block on cerebral vasospasm after subarachnoid hemorrhage in rabbits. Acta cirurgica brasileira. 2013;28(2):89-93.

13. Liu GD, He CJ. Stellate ganglion block promotes recovery of Bell's palsy in patients with diabetes mellitus. Acta Otolaryngol. 2014;134 (6):652-655. doi:10.3109/00016489.2014.880794

14. Feeney DM, Boyeson MG, Linn RT, Murray HM, Dail WG. Responses to cortical injury: i methodology and local effects of contusions in the rat. Brain Res. 1981;211(1):67-77.

15. Lu X, Bao X, Li J, et al. High-frequency repetitive transcranial magnetic stimulation for treating moderate traumatic brain injury in rats: a pilot study. Exp Ther Med.2017;13(5):2247-2254. doi:10.3892/etm.2017.4283

16. Sarioglu Y, Utkan T, Akgun M, Duzcan E, Utkan NZ. Effects of deferoxamine and sympathectomy on endothelin-1-induced contraction and acetylcholine-induced relaxation following subarachnoid hemorrhage in carotid artery. Gen Pharmacol. 1997;28(1):145-151.

17. Starkie RL, Rolland J, Angus DJ, Anderson MJ, Febbraio MA. Circulating monocytes are not the source of elevations in plasma IL-6 and TNF-alpha levels after prolonged running. Am J Physiol Cell Physiol. 2001;280(4):C769-C74. doi:10.1152/ajpcell.2001.280.4.C769

18. Famakin BM, Mou Y, Ruetzler CA, Bembry J, Maric D, Hallenbeck JM. Disruption of downstream MyD88 or TRIF Toll-like receptor signaling does not protect against cerebral ischemia. Brain Res. 2011;1388:148-156. doi:10.1016/j.brainres.2011.02.074

19. Kong Y, Le Y. Toll-like receptors in inflammation of the central nervous system. Int Immunopharmacol. 2011;11(10):1407-1414. doi:10.1016/j.intimp.2011.04.025

20. Desborough JP. The stress response to trauma and surgery. $\mathrm{Br}$ J Anaesth. 2000;85(1):109-117.

21. Catania A, Lonati C, Sordi A, Gatti S. Detrimental consequences of brain injury on peripheral cells. Brain Behav Immun. 2009;23 (7):877-884. doi:10.1016/j.bbi.2009.04.006

22. Loane DJ, Byrnes KR. Role of microglia in neurotrauma. Neurotherapeutics. 2010;7(4):366-377. doi:10.1016/j.nurt.2010.07.002

23. Impellizzeri D, Campolo M, Bruschetta $G$, et al. Traumatic brain injury leads to development of Parkinson's disease related pathology in mice. Front Neurosci. 2016;10:458. doi:10.3389/fnins.2016.00458

24. Lapiz MD, Morilak DA. Noradrenergic modulation of cognitive function in rat medial prefrontal cortex as measured by attentional set shifting capability. Neuroscience. 2006;137(3):1039-1049. doi:10.1016/j.neuroscience.2005.09.031

25. Hilakivi I, Leppavuori A. Effects of methoxamine, and alpha-1 adrenoceptor agonist, and prazosin, an alpha- 1 antagonist, on the stages of the sleep-waking cycle in the cat. Acta Physiol Scand. 1984;120 (3):363-372. doi:10.1111/j.1748-1716.1984.tb07396.x

26. Yang H, Gu ZT, Li L, et al. SIRT1 plays a neuroprotective role in traumatic brain injury in rats via inhibiting the p38 MAPK pathway. Acta Pharmacol Sin. 2017;38(2):168-181. doi:10.1038/ aps.2016.130

27. Zhang XM, Zhang L, Wang G, et al. Suppression of mitochondrial fission in experimental cerebral ischemia: the potential neuroprotective target of p38 MAPK inhibition. Neurochem Int. 2015;90:1-8. doi:10.1016/j.neuint.2015.06.010

28. Sanders RD, Sun P, Patel S, Li M, Maze M, Ma D. Dexmedetomidine provides cortical neuroprotection: impact on anaesthetic-induced neuroapoptosis in the rat developing brain. Acta Anaesthesiol Scand. 2010;54(6):710-716. doi:10.1111/j.1399-6576.2009.02177.x 


\section{Publish your work in this journal}

Neuropsychiatric Disease and Treatment is an international, peerreviewed journal of clinical therapeutics and pharmacology focusing on concise rapid reporting of clinical or pre-clinical studies on a range of neuropsychiatric and neurological disorders. This journal is indexed on PubMed Central, the 'PsycINFO' database and CAS, and is the official journal of The International Neuropsychiatric Association (INA). The manuscript management system is completely online and includes a very quick and fair peer-review system, which is all easy to use. Visit http://www.dovepress.com/testimonials.php to read real quotes from published authors.

Submit your manuscript here: https://www.dovepress.com/neuropsychiatric-disease-and-treatment-journal 